\title{
New method for comparing colour gamuts among printing technologies
}

\author{
E. Perales ${ }^{a}$, E. Chorro ${ }^{a}$, V. Viqueira ${ }^{a}$, F. Martínez-Verdú ${ }^{\mathbf{a}}$, S. Otero ${ }^{b}$ and V. de Gracia ${ }^{b}$ \\ ${ }^{a}$ Department of Optics, University of Alicante, Carretera de San Vicente del Raspeig s/n, 03690, \\ Alicante, Spain \\ ${ }^{\mathrm{b}}$ Technological Institute of Optics, Colour and Imaging AIDO, Nicolas Copérnico n ${ }^{\circ}$ 7, 9, 11 and 13, \\ 46980 Paterna, Valencia, Spain
}

\begin{abstract}
The authors have developed a simple method to compare the colour gamuts of different industries (printing, textiles, plastics, etc.) based on representing the reproduced colours in constant lightness $L^{*}$ and hue $h_{\mathrm{ab}}{ }^{*}$ planes. This method allows the analysis of those aspects related to the comparison between the colour gamuts of different industries and the MacAdam and Pointer limits and also of those aspects related to how the colour solid is filled, whether homogeneously or leaving certain unfilled regions for commercial or design reasons. In particular, the authors have compared the colour gamuts of three printing technologies (electrophotography or laser, inkjet and offset) with the same class of paper and characterisation chart, and in this comparison the laser printer has proved to be the best of three printing devices. On the other hand, the authors have checked that gravure technology is better than the other ones due to the substrate nature used at gravure technology.
\end{abstract}

Keywords: colour gamuts, colour solids, electrophotography, inkjet, offset, gravure

\section{INTRODUCTION}

In colour reproduction, it is generally important for different reasons to know beforehand the colour gamut of reproducible colours. ${ }^{1}$ Some industries applying industrial colorimetry, such as textiles, plastics, leather, paints, usually keep a data base with their colour gamut, which only occasionally, following commercial criteria and fashion, is reproduced in a sampler to allow the customers to judge the colour generating capabilities of the manufacturer. However, few enterprises study whether their colour gamut reaches the MacAdam limits or whether it covers more or less homogeneously the Rösch-MacAdam colour solid. ${ }^{1,2}$ Pointer, in 1980, was one of the first scientists to study this problem. ${ }^{3}$

The MS was accepted for publication on 12 September 2007.

* Corresponding author: Esther Perales, Department of Optics, University of Alicante, Carretera de San Vicente del Raspeig s/n, 03690, Alicante, Spain; email: verdu@ua.es
In 2002, Pointer retook this subject, asking for the collaboration of all persons interested in the matter, to generate a large database of colour gamuts in current industry. ${ }^{4,5}$ The authors are particularly interested in this subject, both in those aspects related to the comparison between the colour gamuts of different industries and the MacAdam limits and in those aspects related to how the colour solid is filled, whether homogeneously or leaving certain void regions.

First, the MacAdam limits are described below. Human colour perception is essentially trivariant in nature. Colours are defined by three parameters: lightness, hue and colourfulness (chroma, purity, saturation, etc.). This means that colours define a three-dimensional structure named colour solid, in which upper and lower vertexes are the absolute or perceptual white and black respectively. The colours shaping the intermediate frontiers, obviously with the maximum colourfulness, are called optimal colours 


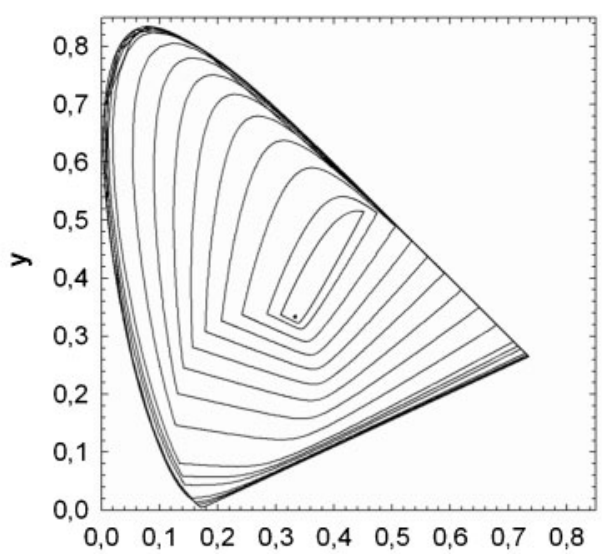

(a)

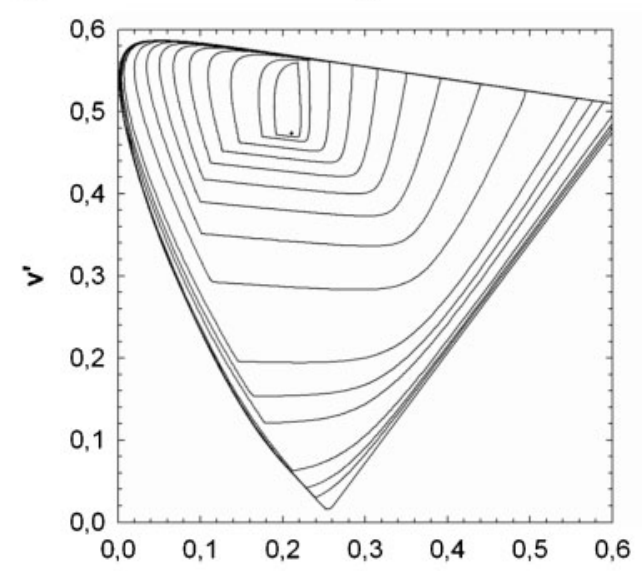

(b)

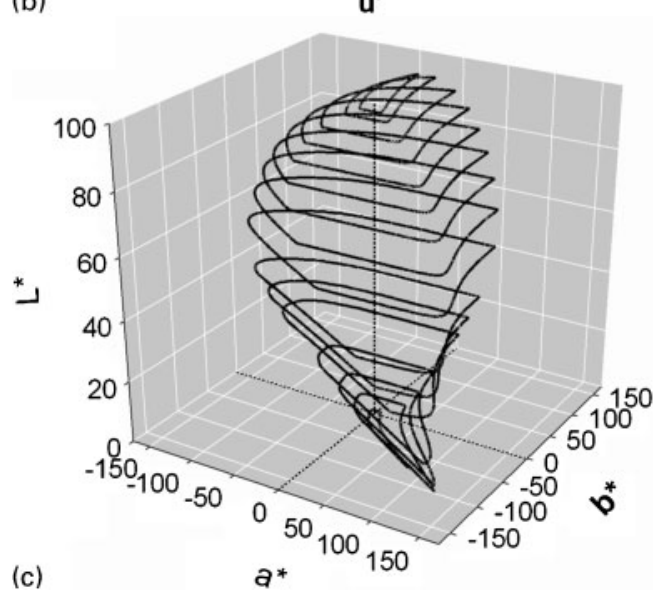

(c)

$a^{*}$

1 MacAdam limits under illuminant $\mathrm{E}$ according to (a) CIE-xy; (b) CIE-u'v' chromaticity diagrams and (c) CIE- $L^{*} a^{*} b^{*}$ colour space

and they were exhaustively studied by MacAdam in 1935. Owing to this, the colour solid borders are also known as MacAdam limits. Rösch in 1929, but above all MacAdam, ${ }^{6,7}$ analysed the theory of optimal colours proving that their spectral reflectance or transmittance can be only zero or one. There are two types of optimal colours (Fig. 1):

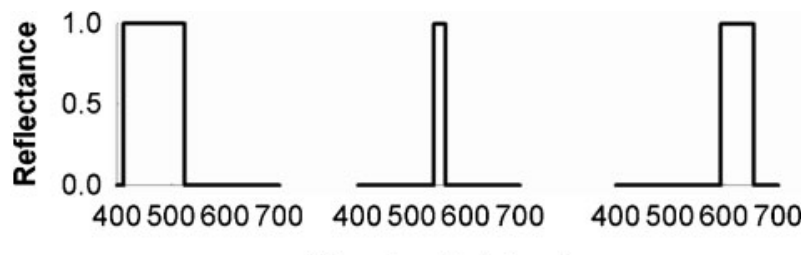

(a) Wavelength $\lambda(\mathrm{nm})$

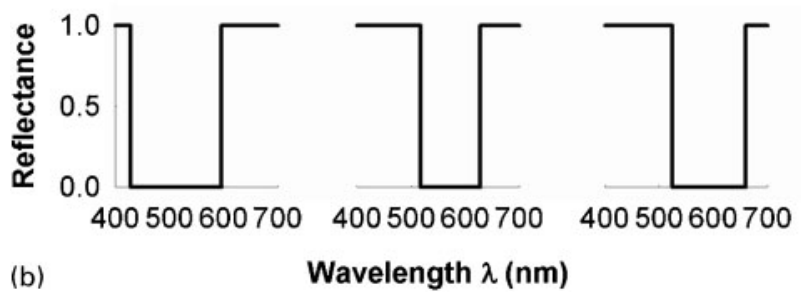

2 Six examples of optimal colours (a) type 1; (b) type 2) with luminance factor $Y=20 \%$ under illuminant $\mathrm{E}$ and CIE-1931 XYZ observer

(i) with 'mountain' like spectral profiles

(ii) with 'valley' like spectral profiles.

As the authors know, although these colours are not present in nature, they are very important for Colour Science because they constitute the frontier of the human colour solid. Therefore, the Rösch-MacAdam colour solid is the colour space derived from the colourmatching functions. ${ }^{2}$ For this reason, the MacAdam limits are used to analyse the colorimetric quality of colorants ${ }^{1,3,4}$ in any industrial application (textiles, paints, printing, etc.). Although there are a lot of artistic attempts and preliminary scientific studies to graph realistically the human colour solid, few exhaustive works have arisen since 1935 based on MacAdam's data. The authors can highlight, as an exception, Pointer's paper from 1980, where different industrial colour gamuts are compared with the MacAdam limits and it is defined the real world surface colour, that is, a limit associated to real colour gamuts of reproduction technologies developed in that moment. Since then, these data (Fig. 2) have been shown sporadically in colour science textbooks, ${ }^{1,2,5}$ but almost always in chromaticity diagrams, as constant luminance factor loci, with the same illuminants (A, C, D65 or E). However, the authors have developed recently a new algorithm ${ }^{8}$ for calculating the MacAdam limits for any lightness $L^{*}$, hue angle $h_{\mathrm{ab}}$ * and illuminant (D50, F2, F7, F11, etc.) or light source (Xe, metal halide, etc.).

On the other hand, in Colour Imaging there are other reasons why the computation of the colour gamut in colour devices is important, particularly the need for controlled colour management in input, 
display and printing devices. ${ }^{9-12}$ Thus, most industries use colour charts to compute the colour profile of colour devices. For many years, for example, the ANSI IT8 charts have served as a reference to calibrate scanners and printing devices. Several works $^{12,13}$ have studied the suitability and the efficiency of these colour charts depending on the device to be characterised. It is for this reason that, for instance, two versions of the ColorChecker chart are available for colour cameras: the classic one and the new DC chart. Similarly, many charts (ECI 2002, CIE 2.9 offset, etc.) offer now an alternative to the ANSI IT8 7/3 chart for printing devices. The aspects taken into account in the design of these colour charts, such as linearisation curves, primary and secondary colours and so on, are those that best characterise the colour device under study. Using these standard colours, the colour gamut of the device can be compared with the MacAdam limits, although few studies have analysed whether the rest of the colours fill homogeneously the RöschMacAdam colour solid. For instance, an exhaustive study of the differences arising from the use of different inks and substrates (paper, cardboard, etc.) in the different printing technologies (flexography, gravure, offset, electrophotography, inkjet, etc.) cannot be found in scientific literature.

For all these reasons, and following Pointer, the authors have developed a simple method to compare the colour gamuts of different printing technologies based on representing the reproduced colours in constant lightness $L^{*}$ and hue $h_{\mathrm{ab}}{ }^{*}$ planes to be compared with the MacAdam limits and the real world surface colour defined by Pointer. In particular, the present work focused on the comparison of four printing technologies, electrophotography or laser, gravure, inkjet and offset, using the same class of paper and approximately the same characterisation chart.

\section{METHODS}

The printing devices selected for this analysis were three laser printers (HP 6600 Indigo, Xerox Docucolor 12 and HP 4600), one inkjet printer (HP 1220), one offset printing press (Heidelberg GTO 52 with Euro Offset) and one gravure printing press (HelioKlischograph K405), with the corresponding genuine cartridge units. Trying to get the most homogenous comparative among these printers the authors used plain paper as substrate $\left(100 \mathrm{~g} \mathrm{~m}^{-2}\right.$ and matte or non-coated appearance), except to the gravure printing device, and the authors decided to print the ECI 2002 cyan, magenta, yellow and black (CMYK) chart for laser, gravure and offset printers. On the other hand, the authors selected to print the TC9.18 red, green and blue (RGB) chart for the inkjet printer in order to benefit themselves from its internal driver model or RGB to CMYK conversion.

When it was possible, the spectral reflectance $\rho(\lambda)$ of the patches of each particular colour chart have been measured by a Minolta CM-2600d spectrophotometer ( $d / 8$ geometry) using the Minolta SpectraMagic 3.6 control software. The tristimulus values $X Y Z$ under illuminant D65 and the CIELAB descriptors $L^{*} a^{*} b^{*} C_{\mathrm{ab}}{ }^{*}{ }_{h} \mathrm{ab}$ are computed along with the $\mathrm{H} \mathrm{V/C} \mathrm{Munsell} \mathrm{descriptors} \mathrm{of} \mathrm{the} \mathrm{sample.} \mathrm{Taking}$ also into account the MacAdam limits under D65 ${ }^{8}$ and Pointer' real world surface colour previously transformed by a chromatic adaptation, as the CAT02 transform of the CIECAM02 colour appearance model, ${ }^{15}$ the authors can select the constant lightness $L^{*}$ and hue $h a b^{*}$ profiles in which the samples of each manufacturer (printing technology) and the MacAdam and Pointer loci must be plotted. In other circumstances, for instance the gravure data, the authors use a GretagMacbeth SpectroScanT spectrophotometer ( $45 / 0$ geometry) to get output data in CIELAB format under illuminant D50 in the corresponding ICC profile.

Once all the patches of each chart/printer are measured in turn, CIELAB data are grouped in parallel ordering them by increasing lightness $L^{*}$ and hue angle hab*. CIELAB data are plotted into constant lightness (luminance factor) planes with a variance of $\Delta L= \pm 5 \%$. On the other hand, the same CIELAB data now ordered by hue are plotted into constant hue angle planes with a variance $\Delta h^{*}$ associated to the hue angle range of the major hues of the Munsell notation (R, YR, Y, GY, etc.). As reference hue angle for each two-dimensional (2D) profile in this case, the authors selected the corresponding value of each Munsell chip with $V=5$ and $C=10$ under illuminant $C$ and according to CIE-1931 observer.

\section{RESULTS AND DISCUSSION}

With this methodology some studies can be conducted: 

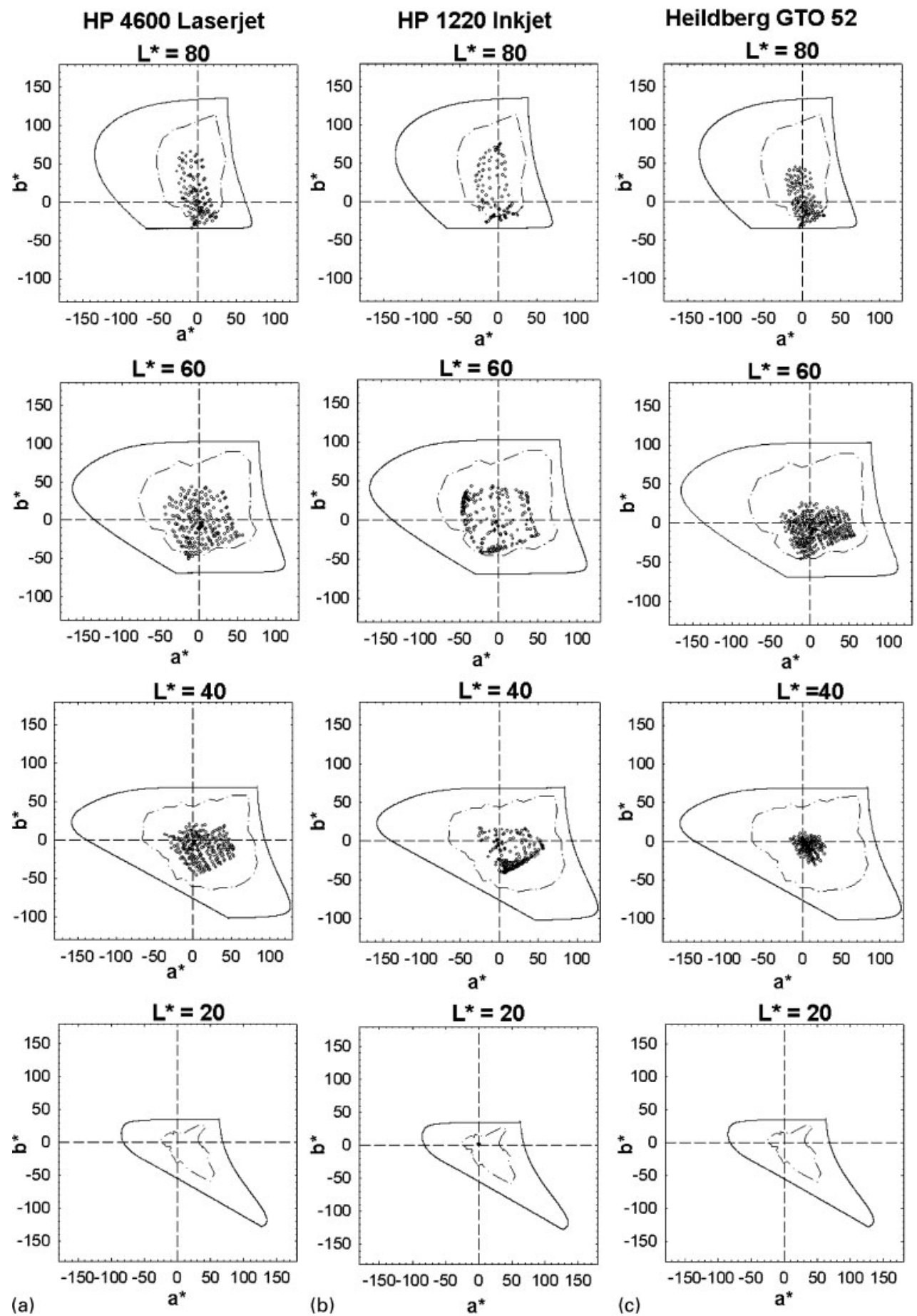

3 (a) samples of ECI CMYK 2002 chart printed in HP 4600 laserjet colour printer; (b) samples of TC9.18 RGB chart in HP 1220C inkjet printer; (c) samples of ECI CMYK 2002 chart printed in Heidelberg GTO 52 printing machine and in several constant lightness planes: outer loci are always corresponding MacAdam limits and the dashed line is for the Pointer's real world surface colour

(i) calculating and graphing the international colour consortium profile data in several constant lightness and hue angle planes (ii) comparing colour gamuts with different categories of paper (uncoated, coated, recycled, etc.) or other substrates in the same printing technology 
HP 4600 LaserJet

5BG - 5R
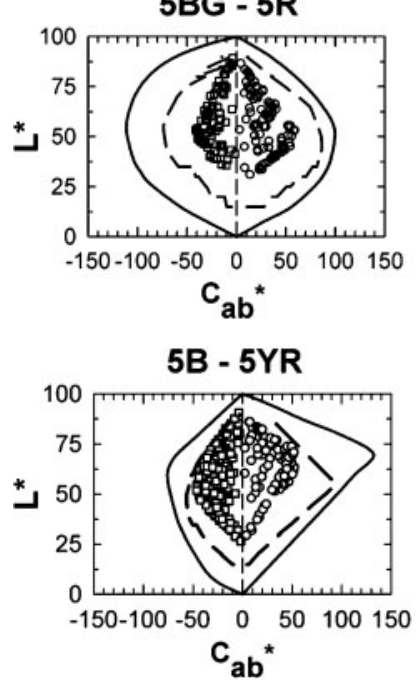

$5 Y-5 P B$

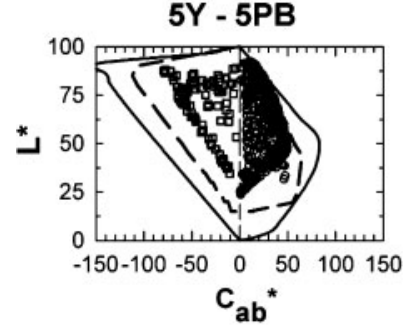

$5 G Y-5 P$
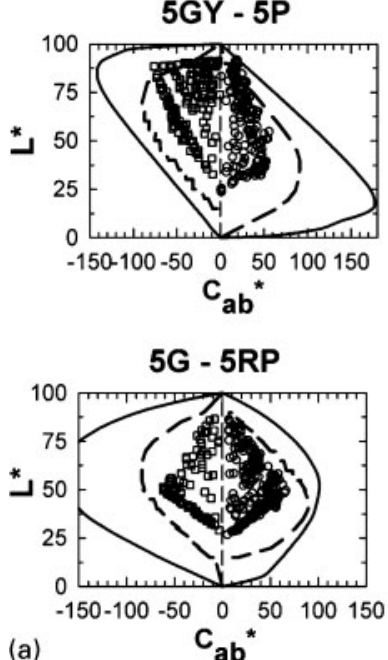

HP 1220 Inkjet 5BG - 5R

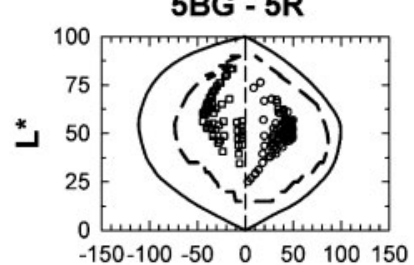

$\mathrm{C}_{\mathrm{ab}}{ }^{*}$

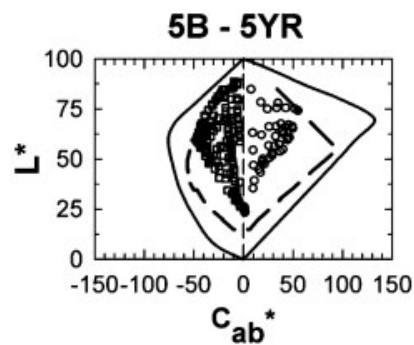

$5 Y-5 P B$

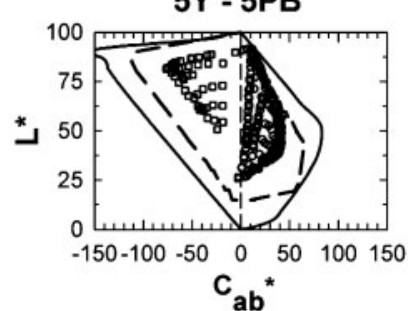

5GY - 5P

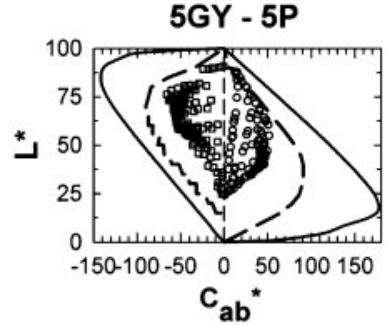

5G - 5RP

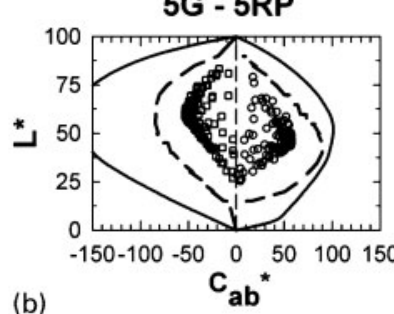

Heildberg GTO 52

5BG - 5R
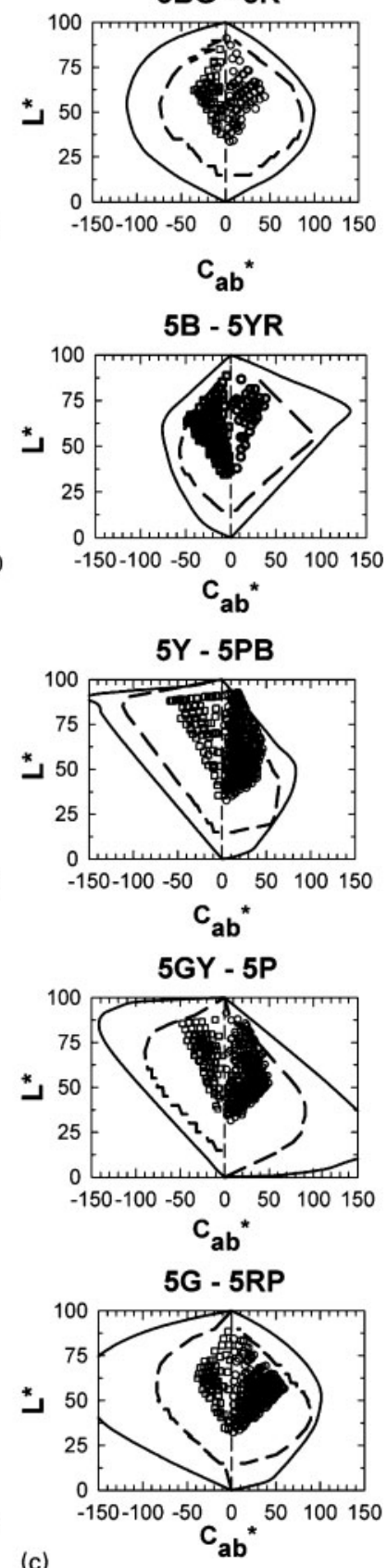

(c)

4 (a) samples of ECI CMYK 2002 chart printed in HP 4600 laserjet colour printer; (b) samples of TC9.18 RGB chart in HP 1220C inkjet printer; (c) samples of ECI CMYK 2002 chart printed in Heidelberg GTO 52 printing machine in several constant hue angle planes: outer loci are always corresponding MacAdam limits and the dashed line is for the Pointer's real world surface colour

(iii) comparing among different CMYK and RGB characterisation charts and to propose improvements to fill more homogenously the Rösch-MacAdam colour solid

(iv) searching new colorants (pigments and inks) in all printing technologies to reach the borders of the MacAdam limits.
Next, the authors show some examples of this methodology.

Comparing among different printing technologies with same paper

Figure 3 shows a comparison between the colour gamuts of the three printers (HP 4600 Laserjet, HP 


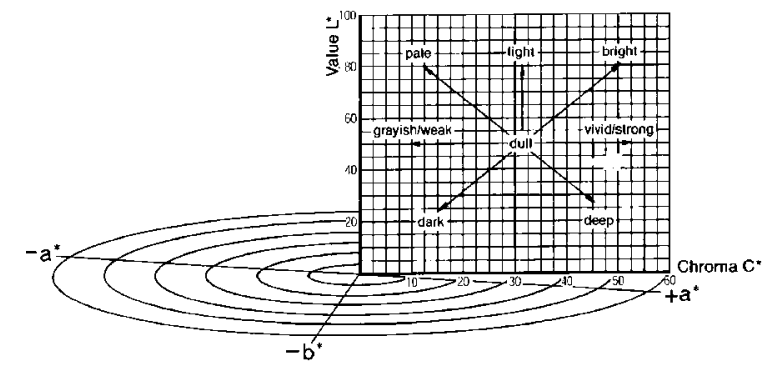

5 Nuance types in constant hue angle section in CIELAB colour space

1220 Inkjet and Heidelberg GTO 52), using the same class of paper, according to several lightness ranges. As it can be seen, none of the three printers reaches the corresponding MacAdam limits, particularly for the lightest colours (section with $L^{*}=80$ ). Furthermore, the colour subgamut of the lightness low range $\left(L^{*}=20\right)$ does not fill the area of the MacAdam loci by any printer. Comparing among the three printers, it is obvious that the best one is the laser printer in the constant lightness profiles from $L^{*}=80$ to $L^{*}=40$. The second place in this ranking is for the inkjet printer, although the colour patches of the TC9.18 RGB do not fill homogenously each subcolour gamut of this printer. Finally, the offset printing machine shows the smallest colour subgamuts. Perhaps using a coated paper, with a grammage $>100 \mathrm{~g} \mathrm{~m}^{-2}$, the colour gamut of this printing device could be significantly increased. The colour gamut obtained with these printers is not much wider than the maximum real world surface colour defined by Pointer in 1980.

On the other hand, Fig. 4 shows the colour gamuts of the same three printers for the Munsell Hue ranges. As it can be seen, none of the three printers reaches again the corresponding MacAdam limits. In general, the blue (B) and blue-purple (PB) MacAdam limits are relatively well filled for the three printers. However, in the other extreme case, the green (G) and purple (P) MacAdam limits are the worst filled by the colour subgamuts of the printers. Also, the authors have checked that the Pointer's real world surface colour is wider than the colour gamut associated to each printer in all hue profiles. Comparing among the three printers, it is again obvious that the best one is the laser printer in the 10 constant hue angle sections. The second place in this ranking is again for the inkjet printer. However, in this type of analysis, it is common for all the printers to leave empty or unoccupied subregions in these hue sections, especially around the achromatic axis, as can be clearly seen in particular with the inkjet printer. Examining each hue section, and taking into account the typical scheme of nuances in constant hue angle profile (Fig. 5), the following can be seen:

(i) there are more deep red colours in laser and inkjet printers than in offset printer

(ii) there are more strong blue colours in the laser printer but there are more bright blue colours in both inkjet and offset printers, etc.

In both cases and generalising, it can be seen that the colour gamut of the laser printer is larger than the rest, above all for dark colours and red hue ranges. The last place of the ranking is occupied by the offset printer. In any case, as it can also be seen, none of the printer colour gamut reaches completely the corresponding MacAdam limits. Moreover, in some specific profiles, both constant lightness and hue angle segments, certain regions inside the colour solid remain empty, particularly around the achromatic axis. The colour gamut reproduced by each printer is not wider than the Pointer's real world surface colour defined 25 years ago although it is supposed that printing technologies have improved in high degree.

\section{Comparing same printing technology with different substrates}

Figure 6 shows the colour gamuts of the same web fed gravure printing press with several substrates or transparent foils for the Munsell Hue ranges. As it can be seen, none of colour subgamuts with printing technology reaches again the corresponding MacAdam limits and the colour gamut is very similar to the Pointer's real world surface, although it is obtained more dark colours in all hue profiles. Comparing with Fig. 4, associated to several printing technologies with paper as substrate, it can be seen clearly that the gravure technology is higher than the other ones (electrophotography, inkjet and offset). The main reason for this significant difference among these printing technologies is the optical nature of the substrate:

(i) paper as reflective medium for the first one

(ii) transparent foil (cellophane, polyethylene, etc.) as transmissive medium for the last one. ${ }^{15,16}$

Nevertheless, this analysis among these printing technologies is not almost right because it is necessary to take into account the optical influence 
Foil 1
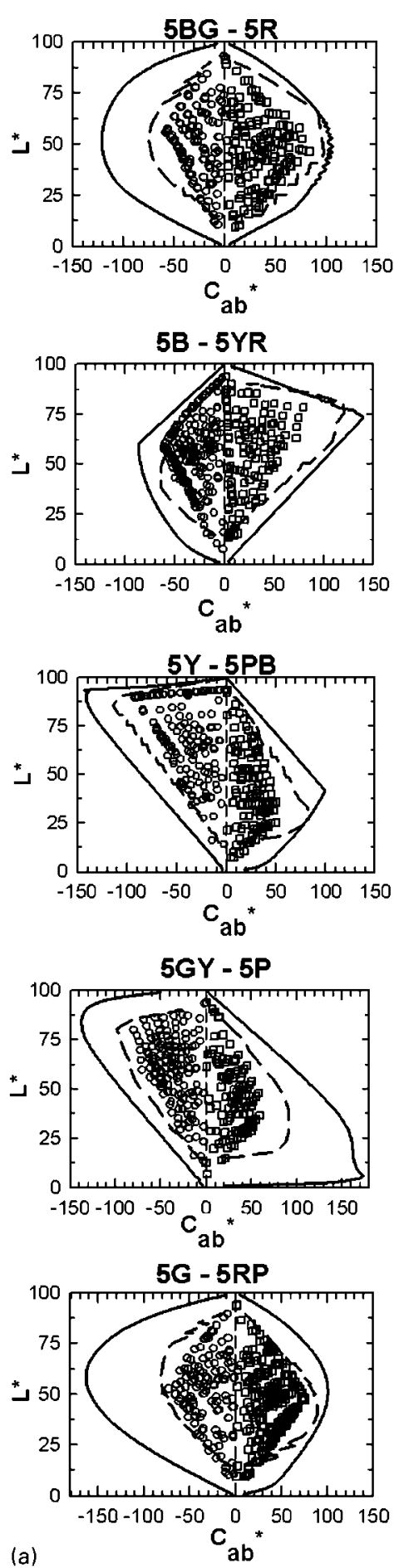

Foil 2
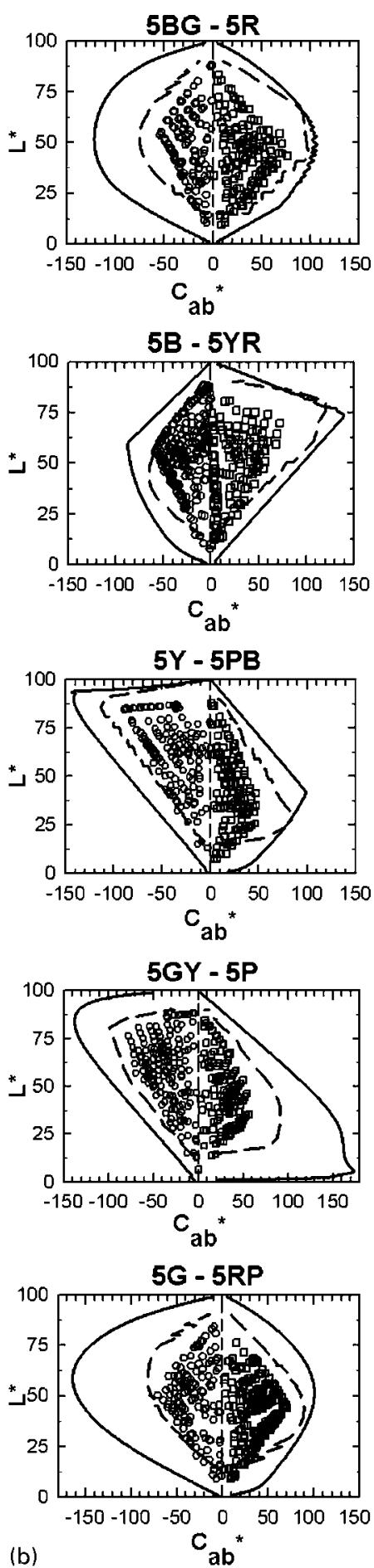

Foil 3

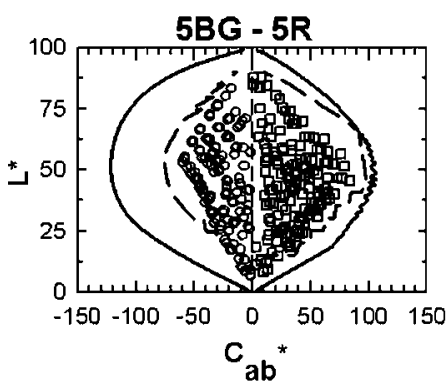

$5 B-5 Y R$
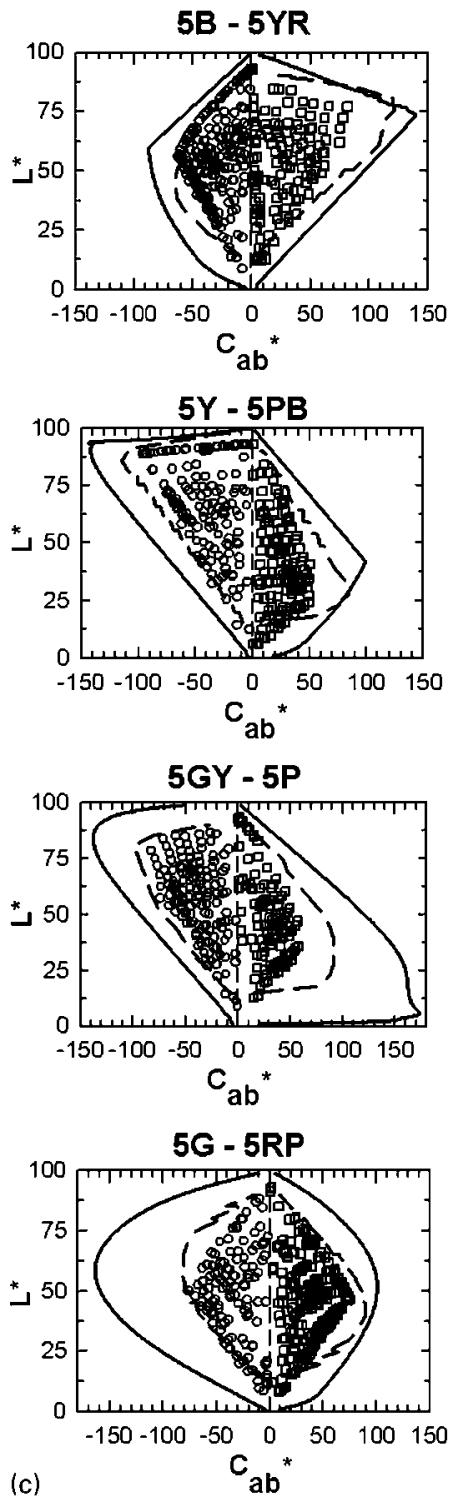

6 Samples of ECI CMYK 2002 chart printed in rotogravure press (HelioKlischograph K405) with several transparent foils in several constant hue angle planes: outer loci are always corresponding MacAdam limits and the dashed line is for the Pointer's real world surface colour

of the reflective substrate of the final packaging materials. ${ }^{15,16}$

In general, all the MacAdam limits are relatively well filled. The worst cases are the green $(G)$ and purple (P) MacAdam limits, but this also was equal in the above printing technologies. Comparing among three transparent foils or substrates, the best one is the foil 1 in the 10 constant hue angle sections. 
Examining each hue section, and taking into account the typical scheme of nuances in constant hue angle profile (Fig. 5), the following can be seen:

(i) there are more deep red colours in foil 3 than in other ones

(ii) there are more strong blue colours in the laser printer but there are more bright blue colours in foil 1 , etc.

However, in this case, the colour gamuts obtained with this printing technology is not much wider either.

\section{CONCLUSIONS}

As an example of the described methodology, which can be applied to any colouration technology (textile, paints, plastics, etc.), the authors compared the colour gamuts of some printing technologies (electrophotography or laser, inkjet and offset) with the same class of paper and characterisation chart (really the ECI 2002 CMYK for the laser and offset printing devices, but the TC9.18 RGB for the inkjet printer). The authors show in this comparison that in general the colour gamut of the laser printer is larger than those corresponding to other printers, as appear from both the constant lightness and the constant hue angle $2 \mathrm{D}$ plots. But whether the authors compare these printing technologies with the gravure printing, they obtain that the gravure printing is better due to the nature substrate used in this printing technology. However, since the authors always include the corresponding MacAdam limits in the figures, and they are almost never reached for the analysed printers, and on the other hand, the Pointer's real world surface is not increased, the authors think that more research is necessary to obtain new dyes and pigments in printing technologies and paper industry for trying to reach the perceptible limits of the human eye. Therefore, the authors think that this new methodology, based on the exhaustive analysis of the colorimetric data in CIELAB colour space, could help to offline quality control in all media print.

\section{ACKNOWLEDGEMENTS}

This research was supported by the Ministerio de Educación y Ciencia (Spain) under grant no. DPI2005-08999-C02-02, and by the Conselleria d'Empresa, Universitat i Ciència of the Generalitat Valenciana (Spain) under grant no. IIARC0/2004/59.
E. Perales would like to thank the Spanish Ministry for Education and Science for the PhD grant that she has received.

\section{REFERENCES}

1 R. S. Berns: 'Principles of color technology', 3rd edn, 143-146; 2000, New York, John Wiley \& Sons.

2 R. G. Kuehni: 'Color space and its divisions: color order from antiquity to the present'; 2003, New York, John Wiley \& Sons.

3 M. R. Pointer. The gamut of real surface colors. Color Res. Appl., 1980, 5, 145-155.

4 M. R. Pointer. Request for real surface colors. Color Res. Appl., 2002, 27, 374.

5 H. Kipphan: 'Handbook of print media: technologies and production methods'; 2001, Berlin, Springer.

6 D. L. MacAdam. The theory of the maximum visual efficiency of colored materials. J. Opt. Soc. Am., 1935, 25, 249-252.

7 D. L. MacAdam. Maximum visual efficiency of colored materials. J. Opt. Soc. Am., 1935, 25, 316-367.

8 F. M. Martínez-Verdú, E. Perales, E. Chorro, D. Fez, V. Viqueira, E. Gilabert. Computation and visualization of the MacAdam limits for any lightness, hue angle and light source. J. Opt. Soc. Am. A, 2007, 24A, 1501-1515.

9 P. Green and L. W. MacDonald: 'Colour Engineering: achieving device independent colour'; 2002, Chichester, John Wiley \& Sons.

10 L. W. MacDonald and M. R. Luo: 'Colour image science: exploiting digital media'; 2002, Chichester, John Wiley \& Sons.

11 B. A. Wandell and D. L. Silverstein: 'Digital color reproduction', 2nd edn, 281-316; 2003, New York, Elsevier.

12 S. Smyth: 'The print and production manual', 9th edn; 2003, Leatherhead, Pira.

13 T. I. V. Cheung and S. Westland. Color Selections for Characterization Charts, Proc. Int. Conf. on 'European conference on colour in graphics, imaging and vision: CGIV'2004', Aachen, Germany, April 2004, Technology Center AGIT, 116-119.

14 CIE 159:2004, 'A colour appearance model for colour management systems: CIECAM02', Commision Internationale de L'Eclaraige, Vienna, 2004.

15 A. Paul and M. Brune: 'Untersuchungen zur Optimierung der Farbwiedergabe bei der frequenzmodulierten Rasterung', FOGRA Forschungsbericht Nr. 52.019, München, 1997.

16 H. Mantler and J. Rodriguez Giles. The Combination of Gravure or some Ink-Jet Screening and the Diffusion of Light in the Substrate Lead to an Experimentally Confirmed Increase of Printable Color Hues. Proc. Int. Conf. on 'Advances in printing and media technology': 32nd IARIGAI, Porvoo, Finland, September 2005, VTT Technical research Centre of Finland, 143-153. 